\title{
Die Entheroisierung des Mose im klassischen Judentum
}

\section{Gabrielle Oberbänsli-Widmer}

Aus Sicht des Alten Testaments mag Mose tatsächlich heroisch anmuten. Schon als Säugling von einem tyrannischen Pharao verfolgt, wird er als junger Mann einen gepeinigten Glaubensgenossen rächen, später sein Volk aus der ägyptischen Knechtschaft in die Freiheit führen, allen inneren und äußeren Anfechtungen auf dem vierzigjährigen Weg durch die Wüste trotzen, Wunder vollführen und - auch das gehört ins stimmige Heldenbild - einen tragischen Tod im Anblick des gelobten Landes sterben.

Im klassischen Judentum, in der spätantiken talmudischen Tradition indes finden sich nur wenige Voten, welche Mose einen Status als Heros attestieren, eine Stelle in der Mischna etwa, dem nachbiblischen Gesetzeskodex, die rhetorisch fragt: „Wer ist größer als Mosche? Mit ihm beschäftigte sich nur Gott ...“. ${ }^{1}$ Sonst aber zeichnet sich im rabbinischen Schrifttum eine unverhohlene Demontage des Exodus-Helden ab. Manchenorts wird er aus der Liturgie verbannt, sein Part am Befreiungsgeschehen gestrichen, seine biblischen Fähigkeiten gestutzt, und - besonders signifikant - sein Name erhält in hebräischen Traditionsschriften ein festes Epitheton: Mosche Rabbenu, Mose, unser Rabbi, Mose, unser Lehrer. Schulstuben aber sind selten Orte, aus denen sich Recken rekrutieren, und Schulmeister üblicherweise kaum der Stoff, aus dem Heroen sprießen. Kurz: Die talmudischen Weisen entziehen dem alttestamentlichen Protagonisten sein heroisches Potential und verengen seine vielfältigen biblischen Rollen auf eine erzieherische Aufgabe im Lehrhaus.

Dem ist nachzugehen. Was treibt die Rabbinen in den ersten nachchristlichen Jahrhunderten zu einem solchen beinahe despektierlichen Unterfangen? Und wie wird es konkret realisiert? Insbesondere angesichts der frühchristlichen und frühjüdischen Rezeption, welche demgegenüber die Mose-Gestalt geradezu messianisch überhöhen. Die rabbinische Entheroisierung des Mose ist mithin ein spannendes und zudem wenig bearbeitetes Feld in der Wirkungsgeschichte dieser für Judentum und Christentum zentralen Figur. Eine Dynamik, in der Sakralität und Heldentum, Heroisches und Religiöses zusammenlaufen und sich in die Realund Mentalitätsgeschichte ihrer Epoche einschreiben.

Dazu eingangs eine Klärung und eine Prämisse.

Die Klärung: Unter ,klassischem Judentum' ist die nachalttestamentliche, spätantike Tradition der talmudischen Rabbinen zu verstehen, das heißt der Weisen,

1 Sota I,9. 
welche die biblisch-israelitische Religion mit ihrer Eigenstaatlichkeit und ihrem Zentralheiligtum nach 70. n. Chr., nach der Zerstörung Jerusalems und des Zweiten Tempels, zu einer gänzlich veränderten Exilsreligion umgeschmiedet haben, deren Strukturen das normative Judentum bis heute nachhaltig prägen.

Die Prämisse: Ohne den geneigten Leser, die geneigte Leserin des biblischen Analphabetismus zu bezichtigen, mögen einleitend ein paar Eckdaten der alttestamentlichen Textvorgabe hilfreich sein, auf deren Grundlage sich die Wirkungsgeschichte dann umso klarer abheben soll.

Auf der Handlungsebene der Bibel ist Mose der dominierende Protagonist der Thora, vom Buch Exodus bis zum Deuteronomium. Orte der Handlung sind Ägypten und die Wüste Sinai. Die Handlungszeit: um 1300 v. Chr. Der Plot: Nachdem die Jakob-Söhne vor Generationen aufgrund einer Hungersnot aus dem Lande Kanaan nach Ägypten ausgewandert sind, fristen die Israeliten nun eine Fronexistenz im Exil, unterdrückt und verfolgt vom Pharao, der die hebräischen Neugeborenen kurzerhand umbringen lässt. Als Säugling überlebt Mose das Massaker nur, weil seine Mutter ihn in einem Körbchen am Nil aussetzt und er wundersam und ausgerechnet von der Tochter des Pharao gerettet wird. Dieses Wandermotiv des im Wasser ausgesetzten Kindes ist weithin bekannt, wobei Exodus 2 eine besondere Affinität zur akkadischen Sargon-Legende aufweist. ${ }^{2}$ Obwohl Mose am Pharaonenhof aufwächst, sieht er die Not des hebräischen Volkes und erschlägt einen ägyptischen Häscher, worauf er fliehen muss. Als Schafhirte in der Wüste erhält Mose in einer göttlichen Berufung den Auftrag, die Israeliten aus Ägypten zu befreien. Sekundiert von seinem eloquenten Bruder Aaron tritt Mose vor den Pharao. Erwartungsgemäß widersetzt sich dieser dem Plan. Erst zehn - die später danach benannten ,ägyptischen' - Plagen zermürben den Despoten. So lässt der Pharao das Volk Israel ziehen, jagt ihm dann aber nach. Doch durch ein Wunder ziehen die Hebräer trockenen Fußes durch das Schilfmeer, während die ägyptischen Verfolger darin umkommen. Zur Erinnerung an den Befreiungszug wird das Pesach-Fest eingesetzt. Nunmehr in der Wüste, am Berg Sinai empfängt das Volk mittels Mose die göttliche Thora: Gesetz, Verfassung und Kultkalender zugleich. Diese rechtlichen und kultischen Passagen bestimmen dann über weite Strecken den Pentateuch, während der Plot seltsam stockt, die Story förmlich in der Wüste versandet - vierzig Jahre für einen Weg von wenigen hundert Kilometern. Das muss man sich schon vergegenwärtigen: Die Distanz zwischen den ägyptischen Fronstädten und dem Süden Kanaans ist - etwa auf schweizerische Verhältnisse übertragen - die zwischen Boden- und Genfersee. Vierzig Jahre! Dabei kämpft Mose unermüdlich. Einerseits gegen äußere Feinde, allen voran gegen die Amalekiter, andererseits aber auch mit dem eigenen Volk, das sich angesichts von monotonem Manna und ewigen Wachteln nach den Fleischtöpfen zurück-

2 Zum Wortlaut der akkadischen Sargon-Legende vgl. W. Beyerlin (Hrsg.), Religionsgeschichtliches Textbuch zum Alten Testament, Göttingen 1975, S. 123-124. 
sehnt und wiederholt von seinem Gott abfällt - berühmt ist die Episode vom goldenen Kalb, welche die fatalen goldenen Kälber der späteren Königszeit antizipiert. ${ }^{3}$ Im Jähzorn zerschmettert Mose da die Gesetzestafeln. Schließlich rebellieren selbst Mirjam und Aaron gegen ihren Bruder Mose, von den levitischen Konkurrenten, bekannt als ,Rotte Korach', ganz zu schweigen. Nach Jahrzehnten endlich an der Grenze zum gelobten Land angekommen, bleibt es Mose versagt, seine Lebensaufgabe zu vollenden. Josua wird das für ihn tun. Mose aber darf die ersehnte Erde nicht betreten, da er für den Ungehorsam seines Volkes haften muss. Hier der Wortlaut der Todesszene, der Schluss des fünften Buches Mose:

„1 Und Mose stieg von den Steppen Moabs auf den Berg Nebo, auf den obersten Gipfel, der gegenüber Jericho lag. Und der Herr zeigte ihm das ganze Land [...]. 5 Und dort starb Mose, der Knecht des Herrn, im Lande Moab auf den Befehl des Herrn [al pi Ado$n a j$ - wörtlich... auf den Mund des Herrn]. 6 Und er begrub ihn im Tal, im Lande Moab gegenüber Beth-Peor. Und niemand kennt sein Grab bis auf diesen Tag. 7 Mose war 120 Jahre alt als er starb. Sein Auge war nicht trübe geworden, und seine Lebenskraft nicht gewichen. [...]. 10 Und es stand von nun an kein Prophet mehr in Israel auf wie Mose, mit dem der Herr von Angesicht zu Angesicht verkehrte, 11 in Bezug auf all die Zeichen und Wunder, mit denen ihn der Herr ausgesandt hatte [...]“.4

Soweit das biblische Narrativ. Ganz anders aber die realgeschichtliche Einschätzung. Eine historische Lektüre muss hier als Advocata Diaboli ein paar kritische Beobachtungen nachtragen. Zeitlich sind Mose und der Auszug aus Ägypten, wie schon gesagt, um 1300 v. Chr. anzusetzen. Leider liegen dazu weder außerbiblische Belege aus dem entsprechenden Zeitraum, noch archäologische Befunde vor, kein Tagebuch des Mose als Sensationsfund aus dem Wüstensand des Sinai. ${ }^{5}$ Erste Verschriftlichungen setzen erst Jahrhunderte später ein, ungefähr im achten vorchristlichen Jahrhundert, also in der Königszeit. Mindestens ein halbes Jahrtausend klafft demzufolge zwischen der Gestalt des Mose und ihrer biographischen Formgebung. ${ }^{6}$ Mose ist mithin vor allem andern eine Erinnerungsfigur. Fraglich, ob sich aus dieser noch ein authentischer Kern herausschälen lässt - am ehesten vielleicht der ägyptische Name Mose. ${ }^{7}$ Verführerisch winkt dann die These, Mose in den Bannkreis der Aton-Religion zu stellen, in jenen ägyptischen Monotheismus unter Amenophis IV Echnaton (um 1364-1347 v. Chr.). Doch gilt es, dieser Verlockung zu widerstehen und eine andere Fährte aufzunehmen. Die Erzählung

3 I Kön 12,28.

4 Dtn 34,1.5-7.10.11. Aus dem Hebräischen von G. O.-W.; falls nicht anders vermerkt, stammen die Übersetzungen auch im Folgenden von der Verfasserin.

5 Zu den Gruppen der sogenannten ,Hapiru' und ,Schazu' vgl. R. G. Kratz, Israel als Staat und Volk, in: Zeitschrift für Theologie und Kirche 97/2000, S. 1-17, insbesondere S. 13-17.

6 E. Otto, Mose. Geschichte und Legende, München 2006; H. Schmid, Die Gestalt des Mose. Probleme alttestamentlicher Forschung unter Berücksichtigung der Pentateuchkrise, Darmstadt 1986; R. Smend, Das Mosebild von Heinrich Ewald bis Martin Noth. Beiträge zur Geschichte der biblischen Exegese, Tübingen 1959.

7 M. Görg, Mose - Name und Namensträger. Versuch einer historischen Annäherung, in: E. Otto (Hrsg.); Mose. Ägypten und das Alte Testament, Stuttgart 2000, S. 17-42. 
des Exodus ist zweifellos ein Exilstext. Aber um welches Exil handelt es sich? Um das ägyptische, oder nicht vielleicht um das babylonische im sechsten vorchristlichen Jahrhundert? Die alttestamentliche Forschung tendiert heute dazu, den Pentateuch immer jünger zu datieren, konkret eben in die exilisch-nachexilische Epoche. Der Exodus als Rückprojektion der babylonischen Unterdrückung und Verbannung in eine ferne ägyptische Vergangenheit. Die Annahme könnte Einiges erklären, etwa weshalb Mose biblisch nahezu alle gesellschaftlich relevanten Rollen einnimmt - Gesetzgeber, Kultvollzieher, Levite, Stratege, Richter, Politiker, Prophet -, aber niemals als König auftritt, hatte doch das Königtum mit der babylonischen Deportation definitiv ausgedient und jedes Prestige verspielt. Einer solchen historischen Hypothese zufolge wäre die Gesetzgebung am Sinai nicht fester Bestandteil und Höhepunkt der Exoduserzählung, sondern vielmehr diente der Mose-Plot als narrative Hülle für das Gesetz, als deren Rahmenerzählung oder Legitimationsgeschichte, die viele Jahrhunderte nach der zeitlichen Verortung der Mose-Figur für dieses Gesetz verfasst worden wäre.

Die Fragen sind kaum zu lösen. Man verharrt hier im Hypothetischen. Und die Wachstumsmodelle der Pentateuch-Forschung nehmen kein Ende. ${ }^{8}$ Als Hintergrund für die nun folgende Rezeption des Mose muss das genügen. Zudem liegt für die Rabbinen jede historische Fragestellung außerhalb ihres Denkhorizonts, gilt für sie doch: „En muqdam u-me'uchar ba-Thora - es gibt kein früher oder später in der Thora". 9 Den talmudischen Weisen zufolge wurde die ganze Halacha, das gesamte Religionsgesetz, schriftliche und mündliche Thora (also auch das talmudische Schrifttum) Mose am Sinai gegeben als zeitübergreifende Wahrheit mit unverrückbarer Gültigkeit bis zum heutigen Tag.

Die nachbiblische Wirkungsgeschichte. Die Literatur zu Mose ist über die Jahrhunderte zu einer imposanten Bibliothek von Monographien und Kommentaren, Historischem und Fiktionalem, Prosa und Lyrik, Oper und Drama gewachsen. Obwohl sich das Korpus kaum noch überblicken lässt, wird im Folgenden versucht, aus den jüdischen Texten eine Typologie herauszuschälen, welche Mose epochenübergreifend auf drei Typen reduziert: den Mose-Messias, den ägyptisierten Mose und - dies der Fokus - den entheroisierten Mose. Zu den beiden ersten Typen nur ein paar Stichworte, zwecks deutlicherer Konturierung des entheroisierten Mose.

In die Kategorie des ersten Typus, des Mose-Messias, fügen sich all die Ansätze ein, die Mose als Träger einer wie auch immer gearteten ersten Offenbarung anerkennen, inzwischen aber eine zweite Offenbarung im Blick haben oder diese in Zukunft erwarten. In der Antike gehören dazu die sogenannten ,rewritten Bibles ${ }^{6}$

8 J. C. Gertz, Tora und Vordere Propheten, in: J. C. Gertz (Hrsg.), Grundinformation Altes Testament, Göttingen ${ }^{4} 2010$ (2006), S. 193-311; E. Zenger, Die Bücher der Tora / des Pentateuch, in: E. Zenger [et al.] (Hrsg.), Einleitung in das Alte Testament, Stuttgart ${ }^{3} 1998$, S. 66-176.

9 Pesachim 6b. 
im Umfeld von Qumran, beispielsweise das apokalyptische Jubiläenbuch, jedoch ebenso neutestamentliche Zeugnisse, besonders bildlich etwa die Verklärungsszene der synoptischen Evangelien, wo Mose von Jesus förmlich überblendet wird. Hier zur Vergegenwärtigung ein paar Verse aus der Fassung des Markus:

„2 Und sechs Tage danach nimmt Jesus den Petrus, den Jakobus und den Johannes mit und führt sie auf einen hohen Berg, sie allein. Da wurde er vor ihren Augen verwandelt, 3 und seine Kleider wurden glänzend, ganz weiß, wie kein Färber auf Erden sie weiß machen kann. 4 Und es erschien ihnen Elija mit Mose, und sie redeten mit Jesus. [...]. 8 Und auf einmal, als sie um sich blickten, sahen sie niemanden mehr bei sich außer Jesus." "10

Der Mose-Messias, der neue Mose als eschatologischer Bote und Träger einer erneuerten Lehre tritt denn auch vorwiegend um die Zeitenwende auf, getragen von apokalyptischer Naherwartung. Eine solchermaßen aktualisierte Mose-Figur steht am Scheideweg von Frühjudentum und Frühchristentum einerseits, gegenüber dem talmudischen Judentum andererseits, denn die spätantiken Rabbinen haben verständlicherweise kein Interesse an konkurrierenden Offenbarungsschriften. In späteren Epochen bis in die gegenwärtigen Tage begegnet man dem Mose-Messias verschiedentlich dann wieder, wenn neue Geistesströmungen einen prestigeträchtigen Ahnherrn benötigen. Im Mittelalter etwa spekulieren die Mystiker über ein konsonantisches Neuarrangement einer kabbalistischen Neo-Thora. In der Neuzeit stilisiert Friedrich Schiller Mose zu einem Proto-Aufklärer, ${ }^{11}$ und im 20. Jahrhundert gestaltet ihn Micha Bin Gorion zum Existentialisten. ${ }^{12}$ Die hebräische Techija-Literatur ${ }^{13}$ macht Mose zum frühen Verkünder des Zionismus, und neuerdings vereinnahmt ihn gar die israelische Frauenbewegung. ${ }^{14}$ Soweit der erste Typus, der Mose-Messias.

Der zweite Typus, der des ägyptisierten Mose, stammt aus der Zeit des Hellenismus. Von Anfang an steht er im Zeichen eines Disputs zwischen Juden und Nichtjuden. Denn Mose wird dann in ägyptisches Leinen, Pallium oder Toga gekleidet, wenn es gilt, das Judentum anzugreifen beziehungsweise es zu verteidigen. Dementsprechend fallen die Debatten um einen wie auch immer gearteten ägypti-

$10 \mathrm{Mk} 9,2-4.8$.

11 F. Schiller, Die Sendung Moses, in: K.-H. Hahn (Hrsg.), Schillers Werke. Nationalausgabe, Bd. 17: Historische Schriften, Erster Teil, Weimar 1970 (1789/1790), S. 377-397.

12 M. J. Bin Gorion, Das Bildnis, in: M. J. Bin Gorion (Hrsg.), Born Judas. Altjüdische Legenden und Volkserzählungen, aus dem Hebräischen von R. Bin Gorion, Frankfurt am Main 1981 (6-bändige hebräische Originalausgabe 1916-1923), S. 12-14.

13 Diese Literatur der nationalen ,Auferstehung' umfaßt die frühen und klassisch-zionistischen Schriften.

14 Zur Veranschaulichung hier die Anfangszeilen der Lyrikerin E. Bat-Zion: „Und wenn ich einst eine Heilige werde so wie Mose am Sinai / vierzig Tage und Nächte / dann zeigt mir Gott ein Buch der Weisung / Geheimnisse und verborgene Rätsel / meine Augen werden das Buch durcheilen [...]“. Zitiert nach E. Bat Zijon, Uchsche-ehje qedoscha - Und wenn ich einst eine Heilige werde, in: M. Shaked (Hrsg.), La-nezach anagnech - I'll play you forever: The Bible in Modern Hebrew Poetry. An Anthology (hebr.), Tel-Aviv 2005, S. 172 (aus dem Hebräischen von G. O.-W.). 
schen Mose ambivalent aus, können sowohl positiv wie negativ besetzt sein. ${ }^{15}$ Offensichtlich brachte der ägyptische Priester Manetho im dritten vorchristlichen Jahrhundert als einer der ersten übelste Berichte in Umlauf: Mose als aussätziger ägyptischer Priester, eine Gefahr für Ägypten, kultisch pervertiert, verstümmelt, vertrieben etc. Manethos Darstellung fand unter griechischen und lateinischen Autoren nicht wenige Nachahmer, allen voran den Grammatiker und Rhetor Apion (im ersten Jahrhundert n. Chr.). Ihm bietet auf jüdischer Seite Flavius Josephus mit „Contra Apionem“ nach Kräften Paroli, indem er Mose auf seine Weise als zeitgenössischen Heros verklärt. Man höre nur, wie Flavius Josephus Mose hellenistisch ausstaffiert:

„An Geistesschärfe übertraf er alle Menschen, die je gelebt haben, und geschickt im Erdenken von Plänen, besaß er auch eine wunderbare volkstümliche Beredsamkeit. Seine Stimmungen beherrschte er in solchem Grade, dass sie in ihm gar nicht vorhanden zu sein schienen, und dass er ihre Namen mehr deshalb, weil er sie bei anderen Menschen sah, als von sich selbst her zu kennen schien. Er war ein vorzüglicher Feldherr und ein Seher, wie kaum ein zweiter, sodass, wenn er redete, man Gott selbst zu hören vermeinte. "16

Angesichts der biblischen Vorlage mag eine solche Charakterisierung nicht wenig verwundern, doch wer sich auf Wirkungsgeschichte einlässt, tummelt sich auf reichlich rechtlosem Terrain, dessen Früchte nicht nur besonders üppig, sondern zuweilen auch äußerst gefährlich gedeihen. Denn in der hellenistischen MoseDebatte geht es um politische Polemik mit unabsehbaren Konsequenzen, konkret: um die Ausschreitungen gegen Juden und Pogrome wie diejenigen im antiken Alexandrien. Das ägyptische Erbe des Mose gärt schließlich über die Epochen weiter - die entsprechenden Positionen hat Jan Assmann in seinem ebenso bekannten wie umstrittenen Buch „Moses der ̈̈gypter“ vor ein paar Jahren referiert. ${ }^{17}$ Das eigentliche Glanzlicht setzt diesem zweiten Mose-Strang indes Thomas Mann mit seiner Erzählung „Das Gesetz“ auf, er wiederum als Verteidiger der israelitischen und jüdischen Werte. Mose hier als Spross einer ägyptischen Prinzessin und eines hebräischen Sklaven modelliert, ringt namentlich um die Niederschrift des Dekalogs, dem unverrückbaren ethischen Urrecht, welches Thomas Mann den Nazis 1943 aus seinem amerikanischen Exil entgegenschleudert. ${ }^{18}$ Mithin ist der Typus des ägyptisierten oder ägyptischen Mose seit der klassischen Antike von

15 G. Oberhänsli-Widmer, Mose in apokalyptischer und jüdisch-hellenistischer Literatur, in: G. Krause / G. Müller (Hrsg.), Theologische Realenzyklopädie, Bd. 23, Berlin 1994, S. 347357; M. Stern, Greek and Latin Authors on Jews and Judaism, Bd. 1, Jerusalem 1974.

16 F. Josephus, Jüdische Altertümer, übers. von H. Clementz, Wiesbaden ${ }^{8} 1989$, S. 250, Buch 4, Kapitel 8.

17 J. Assmann, Moses der Ägypter. Entzifferung einer Gedächtnisspur, München/Wien 1998 (amerikanische Originalausgabe 1997).

18 T. Mann, Das Gesetz, in: T. Mann, Die Erzählungen, Frankfurt am Main 1981, Bd. 2, S. 621-672 (Ersterscheinung in englischer Übersetzung in: The Ten Commandments. Ten Short Novels of Hitler's War against the Moral Code, New York 1943; erste deutsche Ausgabe, Los Angeles 1944). 
Antisemitismen einerseits und jüdischer Verteidigung andererseits überschattet, bis hin zu Sigmund Freuds 1939 publiziertem „Mann Moses“.19

Und damit zum dritten und letzten Typus: dem entheroisierten Mose. Er ist zweifellos der am wenigsten bekannte, sprachlich und methodisch am schwierigsten zugängliche, da Talmudim und Midraschim nach wie vor wissenschaftlich wenig erschlossen sind. Der Bezugsrahmen ist das spätantike, normative oder eben klassische Judentum, exiliert sowohl in Babylonien wie in Eretz Israel selber, vorzugsweise in Galiläa. Hier zunächst ein paar Fakten und Textfragmente, zwecks abschließender Erklärung und Auswertung dieser Befunde.

Mosche Rabbenu, ,Mose, unser Rabbi', mit dieser stereotypen Wortverbindung bezeichnet die jüdische Traditionsliteratur die biblische Figur - wie eingangs erwähnt. Kein Exodusheld, sondern der erste Vertreter der rabbinischen Zunft. Vom alttestamentlichen Mose abgesehen, kommt der Name Mosche in der Spätantike kaum vor, weder in Textzeugnissen, noch auf Grabsteinen. Mit der namentlichen Tabuisierung geht eine inhaltliche Ausgrenzung einher, denn - ganz auffällig - fehlt die Nennung des Mose im zentralen Achtzehn-Gebet, während die Erzväter oder auch König David als Präfiguration des Messias darin bestens positioniert werden. Noch viel mehr jedoch befremdet Moses Absenz in der PesachHaggada, denn wo, wenn nicht in diesem liturgischen Exodus-Singspiel, sollte Mose eine Rolle vergönnt sein? Aber nein, keine Erwähnung, ausdrücklich kein Part für den Protagonisten der Thora. Man achte auf den Wortlaut, da wird deutlich jemand ausgebootet:

„Wajjozi'enu ,Der Ewige führte uns aus Aegypten“(V. B. M. 26,8) - nicht durch Vermittlung eines Engels, nicht durch einen Seraph und nicht durch einen Boten, sondern der Heilige, gepriesen sei Er, in seiner Herrlichkeit selbst, wie es heißt (II. B. M. 12,12): ,Ich werde ziehen durch das Land Aegypten in dieser Nacht. Ich werde schlagen jeden Erstgeborenen vom Menschen bis zum Vieh und an allen Götzen Aegyptens werde ich Strafgerichte üben, ich, der Ewige. - Ich werde ziehen im Lande Aegypten, ich, nicht ein Engel. Ich werde schlagen jeden Erstgeborenen, ich, kein Seraph. An allen Götzen Aegyptens werde ich Strafgerichte üben, ich, kein Bote. Ich, der Ewige, ich bin es, kein anderer." 20

Eine solch augenfällige Ausgrenzung muss wohl dahingehend erklärt werden, dass jüdische Kinder von ihrem ersten Seder-Abend an aus der populären Haggada lernen sollen, dass Gott und nicht Mose ihr Ahnvolk auf wunderbare Weise aus Ägypten errettet habe, damit sich ja nicht - fern sei es - irreführende Verwechslungen zwischen Gott und Moses einstellen könnten wie etwa im obigen Zitat des Flavius Josephus.

Auf diesem Hintergrund erklärt sich so manch talmudisches Motiv, das Mose in nicht allzu vorteilhaftes Licht rückt: Mose als unbedarfter Zuhörer in den hintersten Rängen des Lehrhauses, von Rabbi Aqivas subtilen Thora-Vorträgen restlos

19 G. Oberhänsli-Widmer, Klassiker der jüdischen Literatur: Sigmund Freud, Der Mann Moses oder die monotheistische Religion (1939), in: Kirche und Israel 29,1, 2014, S. 74-89.

20 Zitiert nach Haggada schel Pesach - Die Pessach-Haggadah, übersetzt und erklärt von P. Schlesinger und J. Güns, Tel-Aviv 1971, S. 17-18. 
überfordert; ${ }^{21}$ oder Mose, der sich von Gott drei Wünsche erbeten darf, dessen Einsichten in die göttlichen Erläuterungen dann aber kläglich scheitern. ${ }^{22} \mathrm{Ganz}$ besonders irritieren Moses Wundertaten die Rabbinen, sodass sie diese nicht selten homiletisch zurechtstutzen, beispielsweise die hochdramatische Szene aus der Amalekiter-Schlacht, ${ }^{23}$ welche die Mischna kurzerhand $\mathrm{zu}$ einer Minipredigt domestiziert:

„Und es geschah, wenn Mosche seine Hand erhob, siegte Israel, und wenn Mosche seine Hand sinken ließ, siegte ,Amalek'. Können denn Mosches Hände den Kampf fördern oder den Kampf hemmen? Das will vielmehr sagen, dass die Israeliten, solange sie nach oben blickten und ihr Herz dem himmlischen Vater zu eigen gaben, die Oberhand hatten, sonst aber unterlagen." 24

Am deutlichsten indes zeigt sich die rabbinische Entheroisierung in der Todesszene. Moses Tod hat die jüdische Literatur seit der Antike zu ungezählten Fassungen inspiriert, und vorzugsweise die Midraschim zu Petirat Mosche, zum ,Hinscheiden des Mose', erfreuten sich größter Beliebtheit. ${ }^{25}$ Moses Tod als ausladendes tragikomisches Drama, als munterer Totentanz mit einem denkbar hasenfüßigen Mose und einer nicht minder furchtsamen Mose-Seele, welche verzweifelt mit Gott und Engeln um ein winziges Weilchen Lebenszeit feilschen, bevor der Heilige, gepriesen sei er, Mose endlich mit einem zarten Todeskuss heimzuführen vermag - heißt es doch in Deuteronomium 34,5: ,al pi Adonaj - auf den Mund des Herrn“, rabbinisch eben ein göttlicher Kuss. Hier ein kleines Muster aus dem durchaus heiter arrangierten Sterben:

„Da kam der Heilige, gepriesen sei er, vom allerhöchsten Himmel herab, um die Seele des Mose zu nehmen, und mit ihm die drei Dienstengel: Michael, Gabriel und Sagsagel. Michael breitete das Lager des Mose aus, und Gabriel legte ein Byssuskleid an sein Kopfende, Sagsagel eines an sein Fußende. Michael stand zu der einen, Gabriel zu der anderen Seite. Der Heilige, gepriesen sei er, sagte zu Mose: ,Mose, schließe deine Augen, dein eines wie dein anderes Lid!' Und er schloss seine Augen, das eine wie das andere Lid. Er sagte zu ihm: ,Lege deine Beine aneinander, das eine an das andere!' Er legte seine Beine aneinander, das eine an das andere. In ebendieser Stunde rief der Heilige, gepriesen sei er, die Seele aus seinem Körper."26

Diese Szenerie erinnert wenig an den biblischen Berg Nebo mit dem hünenhaften Mose, vielmehr liegt hier ein alter Mann (immerhin 120-jährig!) auf seinem

21 Menachot 29b.

22 Berachot 7a.

23 Ex 17,11.

24 Rosch Haschana III,8. Zitiert nach Mischnajot. Die sechs Ordnungen der Thora. Hebräischer Text mit Punktation, deutscher Übersetzung und Erklärung, Teil 2: Moed, übersetzt und erklärt von E. Baneth, Basel ${ }^{3} 1986$, S. 404-405.

R. Kushelevsky, Moses and the Angel of Death, New York/Bern 1995; K. Haacker / P. Schäfer, Nachbiblische Traditionen vom Tod des Mose, in: O. Betz [et al.] (Hrsg.), Josephus-Studien. Untersuchungen zu Josephus, dem antiken Judentum und dem Neuen Testament. Otto Michel zum 70. Geburtstag gewidmet, Göttingen 1974, S. 147-174.

Midrasch Devarim Rabba 11,10. 
Krankenlager, ein einfaches ängstliches Menschenkind von fürsorglichen Engeln und einem väterlich-göttlichen Beschützer liebevoll begleitet.

Der rabbinischen Beispiele, die Mose so oder ähnlich auf seine Conditio bumana zurückweisen, wären noch viele. Doch möge das Anschauungsmaterial genügen. Relevant sind die Gründe einer solchen Entheroisierung. Diese hier zum Schluss.

Die Gründe sind sowohl sozio-politisch als auch ideologisch bedingt. Ein erster Grund. Zwar verfassen die Rabbinen einzig religiöse Literatur, aber dennoch verpacken sie zuweilen zeitgeschichtliche Bezüge in ihre Texte, höchst allusiv und fein verschlüsselt versteht sich. ${ }^{27} \mathrm{Da}$ die Midraschim und damit die meisten rabbinischen Mose-Entwürfe in Galiläa, im unmittelbaren Kulturkontakt mit dem erstarkenden Christentum entstehen, nehmen sie zweifellos auch darauf Bezug stets wichtig zu wissen, was die Konkurrenz denkt. Folglich richtet sich der rabbinische Mose gegen die konkurrierende Religion, gegen ein Christentum, welches die Rabbinen als polytheistisch verbrämt wähnen, während das Judentum für sie den uneingeschränkten Monotheismus verkörpert. Ergo ist das Judentum kein Mosaismus, keine nach dem Religionsstifter benannte Mose-Religion, so wie das Christentum die Religion des Jesus als Christus ist, eines Jesus zudem, der als Sohn Gottes verehrt wird. Für die jüdischen Weisen gibt es einen einzigen Helden Israels, nur einen, der Wunder vollbringt und nur einen, der die Israeliten aus Ägypten geführt hat, und das ist Gott, der Heilige, gepriesen sei er. Die neutestamentlichen Wundererzählungen konnten den talmudischen Lehrern kaum entgangen sein, sodass sie deshalb Moses biblische Wunder wie etwa das der Amalekiter-Schlacht nach Kräften redimensionierten. ${ }^{28}$

Ein zweiter Grund. Die Rabbinen vermochten die politische Lage ihrer Gemeinschaft durchaus nüchtern einzuschätzen und wollten nach dem verlorenen Krieg gegen Rom jeden Freiheitsaufstand vermeiden. Spätestens nach der verheerenden Bar-Kochba-Revolte um 135 n. Chr. galt es, jede martialisch-virile Dynamik zu dämpfen. Kein neuer Freiheitskämpfer, kein neuer Mose, kein Abschütteln der erneuten Knechtschaft, vielmehr diplomatisch-diskretes Unterordnen unter die entsprechenden Fremdherrschaften und Gastländer war von nun an für fast zwei Jahrtausende die Devise. Erst am Ende der Zeiten - so die rabbinische Vorstellung - werde der von Gott bestellte Messias dem Exil ein Ende setzen. Doch dieser Messias gehe dann als strahlender Sohn Davids aus dem Stamme Juda hervor, und wohlgemerkt nicht aus Mose und dessen Stamm Levi. Für das exilierte Volk Israel gingen mithin Helden in Stil des biblischen Mose mit Destabilisierung und Gefahr einher.

27 Y. H. Yerushalmi, Zachor: Erinnere Dich! Jüdische Geschichte und jüdisches Gedächtnis, aus dem Amerikanischen von W. Heuss, Berlin 1996 (amerikanische Originalausgabe 1982).

28 Die These, dass vor allem die Pesach-Haggada gezielt Gegentopoi zu den Evangelien lanciere, vertritt insbesondere I. J. Yuval, Zwei Völker in deinem Leib. Gegenseitige Wahrnehmung von Juden und Christen in Spätantike und Mittelalter, aus dem Hebräischen von D. Mach, Göttingen 2007 (amerikanische Originalausgabe 2006). 
Ein dritter und letzter Grund. Mosche Rabbenu - eine solche Typisierung lenkt den Blick vom alttestamentlichen Protagonisten auf dessen gänzlich veränderte Rolle als Thora-Lehrer und vor allem auf das Gesetz, das diese Figur in Händen hält. Nach 70 n. Chr., nach dem Verlust von staatlichem Territorium und zentraler Kultstätte, bedeutete die Thora dem Judentum den einzigen verbleibenden Wert. Und deshalb vermag nunmehr keine heroische Menschengestalt die jüdische Identität zu repräsentieren. Diese erhabene Rolle, die jüdische Identität zu stiften, ist jetzt und über die Epochen dem Heiligen Buch vorbehalten. Auf einem so gearteten Hintergrund versteht sich das rabbinische Mose-Bild wie ein Klappbild: Mose tritt dort und dann in großer Pose auf, wenn er Rabbinen und Judentum personifiziert und die göttliche Thora in Händen hält, ${ }^{29}$ aber jämmerlich in seiner conditio bumana. ${ }^{30}$

Soweit der rabbinisch entheroisierte Mose. Bestimmend bleibt diese Sicht für das Judentum von der Spätantike bis heute. Und sie wirkt auch nach der Aufklärung im säkularen Denken nach. Moderne Urteile, die für ein christlich sozialisiertes Publikum nahezu abschätzig klingen mögen, tun es für Juden oder Israelis weit weniger, ist man mit Mose doch auf familiärem Du. In diesem Sinn sei an dieser Stelle das Schlusswort der ersten und bisher einzigen Premierministerin des Staates Israel das Schlusswort überlassen mit ihrer realpolitisch durchaus berechtigten Kritik an Mose: „Vierzig Jahre in der Wüste“, schnauzte Golda Meir, „nur um uns schließlich an den einzigen Ort im Nahen Osten zu führen, wo es kein Öl gibt“. ${ }^{31}$

29 Wie es die Maimonidischen Glaubensgrundsätze im 12. Jahrhundert dogmatisch beschwören werden: „Ich bin vollkommen überzeugt, daß die ganze Lehre, die sich jetzt in unseren Händen befindet, unserem Lehrer Mosche, Friede sei mit ihm, übergeben worden ist. Ich bin vollkommen überzeugt, daß diese Lehre nicht umgetauscht werden wird und keine andere Lehre kommen wird vom Schöpfer, gelobt sei sein Name“. Zitiert nach Sidur Sefat Emet, mit deutscher Übersetzung von S. Bamberger, Basel 1982, S. 79. Mit dieser ,ganzen Lehre' meint Maimonides die schriftliche wie die mündliche Thora und polemisiert damit sowohl gegen die Offenbarungsschriften von Christen und Muslimen, als auch innerjüdisch gegen ein Ausufern der Kabbala und gegen die Ablehnung der mündlichen (d.h. rabbinischen) Traditionen durch die Karäer.

30 Nicht von ungefähr findet sich dieser Midrasch - auch als eigenständiger Text unter dem Titel Midrasch Petirat Mosche, „Midrasch über den Tod des Moses“, - in demselben Manuskript wie der mit „Moses Größe“ überschriebene (Codex h Paris Nr. 710); vgl. dazu die fünf Moses-Midraschim bei A. Wünsche, Aus Israels Lehrhallen. Bd. 1: Teile 1-3. Kleine Midraschim zur späteren legendarischen Literatur des Alten Testaments, Hildesheim 2013 (Leipzig 1907), S. 61-176, S. 126.

31 Zitiert nach A. Oz / F. Oz-Salzberger (Hrsg.), Juden und Worte, aus dem Englischen von E.-M. Thimme, Berlin 2013 (englische Originalausgabe 2012), S. 234. 